\title{
RETOS PARA EL DIAGNÓSTICO PRECOZ DEL CÁNCER DE PRÓSTATA EN EL PERÚ
}

\author{
Mariela Pow-Sang ${ }^{1, a}$, Marco A. Huamán ${ }^{1, b}$
}

\section{RESUMEN}

\begin{abstract}
La detección temprana del cáncer de próstata en el Perú es muy baja, ya que los pacientes, por lo general, llegan con la enfermedad localmente avanzada o avanzada. No existen campañas de tamizaje del cáncer de próstata que nos permitan detectar esta enfermedad en estadios tempranos. Las tasas de incidencia, según el Registro de Cáncer de Lima Metropolitana, van en aumento, a pesar de existir probablemente un subregistro en nuestro país, al no contar con un registro a nivel nacional que muestre la real magnitud de esta enfermedad. Es imperativo generar programas de prevención para el diagnóstico temprano del cáncer de próstata mediante el examen digito-rectal de la próstata y el dosaje del antígeno prostático específico (PSA) en sangre
\end{abstract}

Palabras clave: Cáncer de próstata; Perú, Incidencia; Mortalidad (fuente: DeCS BIREME).

\section{CHALLENGES TO EARLY DIAGNOSIS OF PROSTATE CANCER IN PERU}

\begin{abstract}
Early detection of prostate cancer in Peru is very uncommon, as patients usually arrive when the disease is locally advanced or advanced. There are no prostate cancer screening campaigns that allow us to detect this disease in early stages. The incidence rates, according to the Registry of Cancer in Metropolitan Lima, are increasing. However, there is probably an under register of cases in our country, since there are not any nation-wide records showing the real magnitude of this disease. It is imperative to develop prevention programs for the early diagnosis of prostate cancer through digital rectal exams and to perform the measurement of the prostate- specific antigen (PSA) in the blood.
\end{abstract}

Key words: Prostatic neoplasms; Peru; Incidence; Mortality (source: MeSH NLM).

\section{INTRODUCCIÓN}

Tanto a nivel mundial como nacional, los casos de cáncer de próstata se han incrementado por el aumento de la expectativa de vida de la población. El presente trabajo tiene la finalidad de mostrar el incremento en la incidencia del cáncer de próstata en el Perú desde la década de los setenta hasta la actualidad. Asimismo, demostrar que los pacientes con el diagnóstico de cáncer de próstata llegan a los centros especializados en estadios localmente avanzados o avanzados. Este hecho implica un problema de salud pública por el alto costo del tratamiento en estos estadios y la disminución de la sobrevida.

\section{MAGNITUD DEL PROBLEMA}

El cáncer de próstata tiene una mayor incidencia en varones por arriba de los 50 años de edad, rara vez es diagnosticado en menores de 40 años. La detección es posible con una prueba sanguínea, el antígeno prostático específico (PSA por sus siglas en inglés), y el examen dígito-rectal de la próstata; el diagnóstico se realiza mediante la biopsia transrectal de la próstata bajo guía ecográfica.

A nivel mundial, el cáncer de próstata es el segundo cáncer más diagnosticado en hombres (899 000 nuevos casos al año, $13,6 \%$ del total). Aproximadamente el $75 \%$ de los casos se registran en países desarrollados. Las tasas de incidencia más altas se observan en Australia/Nueva Zelandia (104,2 por 100 000), Europa y Norteamérica, debido al uso del PSA, y a la subsecuente biopsia de próstata, utilizada ampliamente en dichas regiones. Las tasas de incidencia son relativamente altas en algunas regiones en desarrollo como el Caribe, América del Sur y África. La incidencia más baja se observa en Asia (4,1 por 100 000) (1).

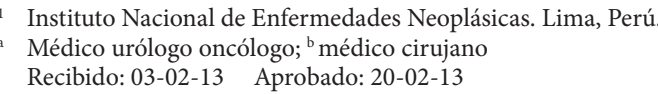


La mortalidad por cáncer de próstata en los Estados Unidos presenta una disminución gradual y sostenida de aproximadamente $30 \%$ (2); esta tendencia se observó poco después de la introducción de la prueba del PSA a fines de la década de 1980; existe evidencia, que el tamizaje con el PSA ha cumplido un rol importante en este sentido ${ }^{(3)}$. El Estudio Europeo Randomizado de Tamizaje del Cáncer de Próstata ha demostrado una reducción relativa del $20 \%$ de las muertes producidas por cáncer de próstata en aquellos que fueron tamizados comparados a los que no lo fueron a 9 años ${ }^{(4)}$.

Previa a la evaluación del estado del cáncer de próstata en el Perú, es importante mencionar que en un país con una población masculina mayor de 50 años reducida, tendrá una baja incidencia de cáncer de próstata. De acuerdo con los censos realizados por el Instituto Nacional de Estadística e Informática en los años 1993 y 2007, se observó que en el año 1993 la población menor de 20 años era aproximadamente $30 \%$ y la mayor de 50 años $7 \%$; mientras que en el 2007 fue aproximadamente 21 y $10 \%$, respectivamente. Asimismo, la expectativa de vida aumentó, en tanto que la tasa de mortalidad infantil disminuyó a lo largo de estos años ${ }^{(5)}$.

En el Perú, el cáncer de próstata presenta una incidencia que va en aumento, asociado al incremento de la esperanza de vida y la longevidad. A la fecha, el Registro de Cáncer de Lima Metropolitana ha publicado cuatro reportes sobre el cáncer en el Perú (6-9). Según este Registro, en el período 1990-1993 los cánceres más frecuentes en varones fueron estómago en primer lugar, seguido de próstata y pulmón, con una tasa de incidencia por 100000 habitantes de 13,$85 ; 12,76$ y 9,82, respectivamente ${ }^{(8)}$. Para 1994-1997, el cáncer más frecuente en Lima Metropolitana fue el de próstata, seguido de estómago y pulmón, con una tasa de incidencia de 18,08; 16,55 y 11,24 por 100000 habitantes, respectivamente (9). Para los años 2004-2005, el cáncer de próstata presentó una tasa de incidencia cruda (TIC) de 34,31 y una tasa de incidencia estandarizada por edad (TIEE) de 35,89 (Figura 1). En Trujillo (1996-2002) la TIC es de 16,0 y una TIEE de 25,8. Finalmente, en Arequipa (2002-2007) presenta una TIC de 35,0 y una TIEE de $37,8^{(10)}$.

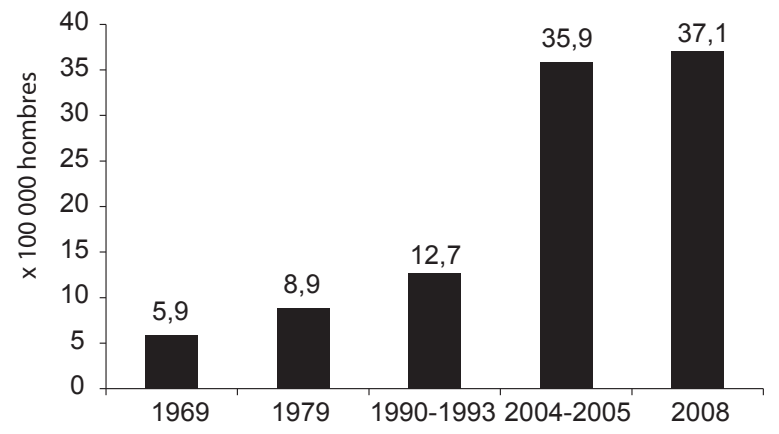

Figura 1. Tasas de Incidencia del cáncer de próstata en el Perú, de acuerdo a los reportes del Registro de Cáncer de Lima Metropolitana y de GLOBOCAN 2008.

El proyecto GLOBOCAN proporciona estimados de la incidencia, mortalidad y prevalencia de los cánceres más frecuentes de 184 países a nivel mundial. El último reporte de GLOBOCAN es del año 2008. Este Proyecto reporta que para el Perú se estimó que en el 2008 ocurrieron 4142 casos nuevos de cáncer de próstata ${ }^{(1)}$. Esa misma fuente reporta que el cáncer más frecuente es el de próstata, seguido de estómago y luego pulmón; con una tasa de incidencia estandarizada por edad de 37,1 ; 22,6 y 7,8 respectivamente. La tasa de incidencia cruda (TIC) del cáncer de próstata fue 28,7 casos por cada 100000 hombres (Tabla 1) y la TEE de mortalidad de $14,0^{(1)}$.

Una revisión de los casos diagnosticados de cáncer de próstata en el Instituto Nacional de Enfermedades Neoplásicas (INEN) en Lima, Perú, entre 1981 y 1985 , revela que el $5 \%$ correspondió a los estadios I y II (enfermedad localizada); el $13 \%$ al estadio III (enfermedad localmente avanzada), y el $76 \%$ al estadio IV (enfermedad metastásica). A través de los años, el diagnóstico de enfermedad avanzada ha ido disminuyendo, mientras que la enfermedad localizada se ha incrementado discretamente. En el último quinquenio, entre el 2006 y el 2010 , el $21 \%$ fueron estadio I-II; $42 \%$ estadio III, y $37 \%$ avanzados (metastásicos) (Figura 2). En los Estados Unidos, el uso generalizado del análisis del PSA ha dado lugar a una migración significativa del estadio y el grado del

Tabla 1. Incidencia, mortalidad y prevalencia a cinco años para el cáncer de próstata en el Perú - GLOBOCAN 2008.

\begin{tabular}{|c|c|c|c|c|c|c|c|c|c|}
\hline \multirow{2}{*}{ Cáncer } & \multicolumn{3}{|c|}{ Incidencia } & \multicolumn{3}{|c|}{ Mortalidad } & \multicolumn{3}{|c|}{ Prevalencia 5 años* } \\
\hline & N. ${ }^{\circ}$ & $(\%)$ & TIEE & N. ${ }^{\circ}$ & $(\%)$ & TIEE & N. ${ }^{\circ}$ & $(\%)$ & Prop. \\
\hline Próstata & 4142 & $(25,1)$ & 37,1 & 1646 & $(15,0)$ & 14,0 & 12034 & $(34,8)$ & 121,0 \\
\hline Estómago & 2593 & $(15,7)$ & 22,6 & 2242 & $(20,4)$ & 19,5 & 4381 & $(12,7)$ & 44,0 \\
\hline Pulmón & 888 & $(5,4)$ & 7,8 & 873 & $(7,9)$ & 7,7 & 855 & $(2,5)$ & 8,6 \\
\hline
\end{tabular}

Incidencia y mortalidad para todas las edades. *Prevalencia a 5 años solo para la edad adulta.

TIEE: tasa de incidencia estandarizada por edad, por 100000 hombres.

Prop: proporción por 100000 hombres 


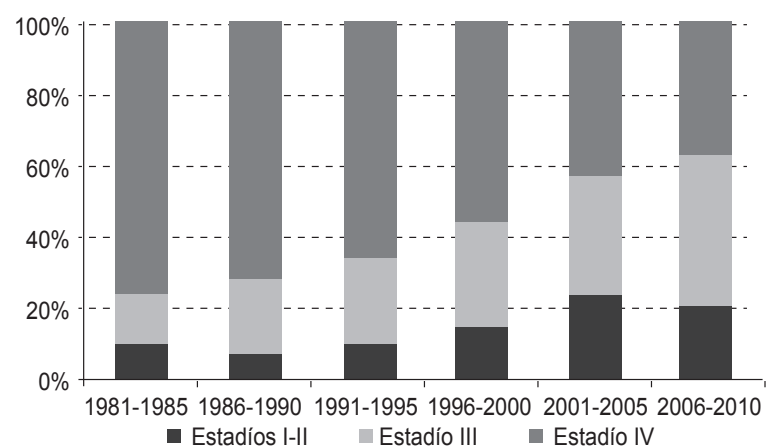

Figura 2. Porcentaje de pacientes con cáncer de próstata diagnosticados en el Instituto Nacional de Enfermedades Neoplásicas, Lima - Perú, por estadios y quinquenios (1981 -2010 ).

cáncer de próstata, de modo que, en la era actual, más del $80 \%$ de los varones son diagnosticados de una enfermedad localizada ${ }^{(11,12)}$.

En el periodo 2006-2010, se reportaron 27 casos (1\%) en menores de 50 años, en la sexta década $12 \%$, en la séptima década $37 \%$, en la octava década 40 , y $10 \%$ en mayores de 80 años. De otro lado, 256 pacientes fueron sometidos a prostatectomía radical en el INEN. El estadio patológico de estos pacientes fue pT0 1\%; pT2 (enfermedad localizada) 51\%; pT3 (enfermedad localmente avanzada) 38\%, y pN1 (enfermedad metastásica ganglionar) $10 \%$. Estos resultados demuestran que aún seguimos diagnosticando un gran porcentaje de pacientes con enfermedad localmente avanzada, a pesar de tener clínicamente la enfermedad localizada.

Considerando que son solo datos del INEN, es probable que el diagnóstico de la mayoría de los casos de cáncer de próstata en estadios avanzados no cambie a nivel nacional, ya que el INEN es el hospital de referencia nacional, al cual acuden pacientes con alta sospecha de cáncer de próstata ${ }^{(13)}$.

\section{COSTOS EN EL TRATAMIENTO DE CÁNCER DE PRÓSTATA}

El tratamiento del cáncer de próstata es considerado un problema de salud pública debido al costo elevado del manejo de los pacientes con enfermedad localmente avanzada o avanzada, y al alto impacto económico que genera en sus familias. El Plan Esperanza, promulgado recientemente por el Estado peruano ${ }^{(14)}$, es un programa que permite la cobertura financiera de los pacientes sin recursos económicos, con los diagnósticos de cáncer de próstata, de colon, de estómago, de mama y de cérvix; así como de leucemias y linfomas. Los gastos generados por la atención de estas enfermedades serán financiados por el Fondo Intangible Solidario de Salud (FISSAL), Unidad Ejecutora 002 del Seguro Integral de Salud ${ }^{(15)}$.

Se ha estimado que el costo promedio del tratamiento de los pacientes tratados en el INEN, con cáncer de próstata localizado sometidos a prostatectomía radical con fines curativos es de S/. 5 357. Sin embargo, cuando el cáncer está localmente avanzado, el costo promedio de tratamiento por año es de S/. 10300 , y si es enfermedad avanzada es entre S/. 31000 y S/. 34 000. Estos costos aumentan considerablemente si el paciente tiene que recibir quimioterapia o tratamiento del dolor en una enfermedad avanzada.

\section{PROBLEMAS EN EL DIAGNÓSTICO}

EI PSA es una proteasa producida por el epitelio prostático normal y maligno, el cual es secretado al líquido seminal. Solo pequeñas cantidades de PSA pasan a la circulación desde el tejido prostático normal, pero aumenta en presencia de enfermedad prostática. La sensibilidad y la especificidad del PSA por arriba de $4 \mathrm{ng} / \mathrm{mL}$ son del 20 y del 60 a $70 \%$, respectivamente ${ }^{(16)}$. Una manera de mejorar la sensibilidad del PSA es considerar los valores del PSA de acuerdo a la edad. Es así que para hombres entre 40 y 49 años el PSA debe ser menor de $2,5 \mathrm{ng} / \mathrm{mL}$; entre 50 y 59 años menor de $3,5 \mathrm{ng} / \mathrm{mL}$; entre 60 y 69 años menor de $4,5 \mathrm{ng} / \mathrm{mL}$, y mayores de 70 años menor de $6,5 \mathrm{ng} / \mathrm{ml}{ }^{(17)}$. Por otro lado, la velocidad del PSA (VPSA) considera riesgo a desarrollar cáncer de próstata si hay un aumento de $0,75 \mathrm{ng} / \mathrm{mL}$ o más por año del PSA, en varones con PSA entre 4 y $10 \mathrm{ng} / \mathrm{mL}{ }^{(18)}$. Recientemente un estudio sugiere que una VPSA de $0,4 \mathrm{ng} / \mathrm{mL}$ por año puede mejorar la detección del cáncer de próstata ${ }^{(19)}$. El índice de PSA(PSA libre/PSA total) es la prueba más utilizada para discriminar entre hiperplasia benigna de próstata y cáncer de próstata, en varones con PSA entre 4 y $10 \mathrm{ng} / \mathrm{mL}$ y un tacto rectal normal. En un estudio prospectivo multicéntrico se encontró cáncer de próstata en $56 \%$ de hombres con un índice de PSA $<0,10$, pero en solo $8 \%$ de hombres con índice $>0,25$ (20).

Si bien las principales herramientas para el diagnóstico de cáncer de próstata son el tacto rectal y el PSA, en el Perú y en la mayoría de los países de Latinoamérica, la idiosincrasia del varón genera resistencia para realizarse el examen del tacto rectal por la idea prejuiciosa que podría inducirlos al homosexualismo. Asimismo, en las áreas rurales, el sistema de salud pública no cuenta con personal entrenado ni con los medios necesarios para realizar el tacto rectal y la medición del PSA. Estas son algunas de las razones 
por qué se diagnostica el cáncer de próstata en estadios avanzados.

Además, las políticas de salud preventiva no se han llevado adecuadamente, y la población desconoce la ventaja de la prevención de esta enfermedad con el simple hecho de realizarse el tacto rectal y la medición de PSA a partir de los 50 años y no esperar hasta los 60 o los 70 años para realizarse los exámenes al presentar molestias urinarias debido al crecimiento prostático, asociado algunas veces a dolor óseo, lo que sugeriría la presencia de cáncer metastásico. Un estudio prospectivo entre 6630 varones, evalúo la eficacia del tacto rectal y el PSA para la detección temprana del cáncer de próstata, se realizó en este grupo la biopsia de próstata bajo guía ecográfica. Los resultados mostraron que el $15 \%$ de los hombres tenía solo PSA mayor a $4 \mathrm{ng} / \mathrm{mL} ; 15 \%$ tacto rectal sospechoso, y $26 \%$ tanto el PSA como el tacto rectal alterados. De 1167 biopsias realizadas, se detectó el cáncer en $22,6 \%$. La tasa de detección para el tacto rectal fue $3,2 \%$, para el PSA $4,6 \%$ y para la combinación de tacto rectal y PSA $5,8 \%$. Estos hallazgos confirman que tanto el tacto rectal como el PSA se complementan, no siendo suficiente solo el uso de PSA, ya que habrá un $15 \%$ de varones que tendrán el PSA normal y el tacto rectal anormal. De ahí la importancia de realizar la prueba del tacto rectal ${ }^{(21)}$. Un metaanálisis evaluó la eficacia del tacto rectal y la medición del PSA para la detección temprana del cáncer de próstata en varones de 50 años o más, asintomáticos, la detección del cáncer de próstata fue del $1,8 \%$. De los cánceres de próstata detectados, el $83,4 \%$ fue enfermedad localizada ${ }^{(22)}$. Contrariamente, en el INEN, 335 varones que acudieron por alta sospecha de cáncer de próstata fueron sometidos a estas dos pruebas, detectándose el cáncer en 133 (39,7\%). De estos 133 pacientes, 43\% tuvieron enfermedad localmente avanzada y $25 \%$ enfermedad avanzada ${ }^{(13)}$.

\section{RETOS Y DIFICULTADES}

En los últimos meses el Estado peruano ha priorizado el manejo del cáncer desde la prevención, detección temprana y diagnóstico, hasta el tratamiento en todo el país; en este programa está incluido el cáncer de próstata. EI INEN, como órgano rector, está realizando las alianzas estratégicas con los gobiernos regionales, municipalidades, Ministerio de Educación y Ministerio de Trabajo, además de la capacitación del personal de salud del primer nivel de atención, en prevención y diagnóstico temprano en las 24 regiones. Este consta de la capacitación del personal médico en la realización del tacto rectal y la implementación en los centros de salud del dosaje del PSA; además, del reforzamiento de las redes de salud de Lima Metropolitana, Lima Provincias,
Callao, las municipalidades a través de los preventorios, las unidades oncológicas, las instituciones hermanas, organismos no gubernamentales, y la descentralización del diagnóstico y manejo del cáncer en los institutos regionales de enfermedades neoplásicas del norte y sur, con proyección al instituto del centro y del oriente. El principal reto es concientizar a la población masculina para el chequeo temprano a través de la realización de campañas para la detección temprana del cáncer de próstata, y disminuir la morbimortalidad y los gastos económicos por esta causa en los próximos 5 o 10 años. Otro reto es aumentar el diagnóstico de los estadios localizados y realizarles el tratamiento oportuno, especialmente en la población de pobreza extrema quienes, por su condición socioeconómica, no pueden acceder a los sistemas de salud para el tratamiento respectivo. Finalmente, otro reto es promover el despistaje del cáncer de próstata en los exámenes anuales prevacacionales no solo por las empresas privadas, sino también por el sector público.

\section{CONCLUSIÓN}

En resumen, se puede decir que la detección temprana del cáncer de próstata en el Perú es muy baja, ya que la mayoría de los pacientes son diagnosticados con enfermedad localmente avanzada o avanzada, las campañas de tamizaje del cáncer de próstata con el uso del PSA y del examen digito-rectal de la próstata son esporádicas, lo que limita la detección de esta enfermedad en estadios tempranos. Las tasas de incidencia y de mortalidad van en aumento, existiendo un subregistro al no contar con un reporte estadístico a nivel nacional que muestre la incidencia real. El cáncer de próstata es una realidad en nuestro medio y va a ser más frecuente en el futuro mediato debido al incremento de personas adultas mayores. Los resultados mostrados determinan un desafío para la gestión de salud en el marco de la epidemiología del cáncer y de las enfermedades no transmisibles en el Perú. Finalmente, el reto es implementar los programas de prevención para el diagnóstico temprano del cáncer de próstata mediante el examen digito-rectal de la próstata y el dosaje de PSA en sangre a nivel nacional en todos los niveles de atención, así como evaluar los programas de promoción de la salud que se vienen desarrollando desde el año 2011.

Contribuciones de autoría: MPSG y MHC han participado en la concepción del artículo, la recolección de datos, su redacción y aprobación de la versión final. Además, MPSG realizó el análisis e interpretación de datos.

Conflictos de interés: los autores declaran no tener ningún conflicto de interés. 


\section{REFERENCIAS BIBLIOGRÁFICAS}

1. Ferlay J, Shin HR, Bray F, Forman D, Mathers C, Parkin DM. Globocan 2008. V2.0, Cancer incidence and Mortality Worldwide: IARC Cancer Base No 10 (Internet). Lyon, France: International Agency for Research on Cancer; 2010.

2. Ries LAG, Melbert D, Krapcho M, Stinchcomb DG, Howlader N, Horner MJ, et al. SEER Cancer statistics review, 1975-2005 [Internet]. Bethesda: National Cancer Institute [citado el 22 de diciembre del 2012]. Disponible en: http://seer.cancer.gov/csr/1975_2005/

3. Etzioni R, Tsodikov A, Mariotto A, Szabo A, Falcon S, Wegelin J, et al. Quantifying the role of PSA screening in the US prostate cancer mortality decline. Cancer Causes Control. 2008;19(2):175-81.

4. Schröder FH, Hugosson J, Roobol MJ, Tammela TL, Ciatto S, Nelen V, et al. Screening and prostate-cancer mortality in a randomized European study. N Eng J Med. 2009;360(13):1320-8.

5. Perú, Dirección Técnica de Demografía e Indicadores Sociales, Instituto Nacional de Estadística e Informática (INEI). Censos Nacionales 2007: XI de Población y VI de Vivienda. Lima: INEI; 2008.

6. Galvez J. Programa de Registro de Incidencias de Cáncer en Lima Metropolitana. Lima: Instituto de Enfermedades Neoplásicas; 1973.

7. Olivares, J. Cancer incidence in Peru. Proceedings of the First UICC Conference on Cancer Prevention in Developing Countries. The University of Nagoya Press. Nagoya, 1982.

8. Centro de Investigación en Cáncer "Maes-Heller". Registro de Cáncer de Lima Metropolitana. Lima: Instituto de Enfermedades Neoplásicas; 1998.

9. Centro de Investigación en Cáncer "Maes-Heller". Registro de Cáncer de Lima Metropolitana 1994-1997. Lima: Instituto de Enfermedades Neoplásicas; |2004.

10. Perú, Instituto Nacional de Enfermedades Neoplásicas, Departamento de Epi- demiología y Estadística del Cáncer. Informe Ejecutivo: Registro de Cáncer de Lima Metropolitana 2004-2005. Lima: INEN; 2011.

11. Makarov DV, Trock BJ, Humphreys EB, Mangold LA, Walsh PC, Epstein JI, et al. Updated nomogram to predict pathologic stage of prostate cancer given prostate-specific antigen level, clinical stage, and biopsy Gleason score (Partin tables) based on cases from 2000 to 2005. Urology. 2007;69(6):1095-101.

12. Howlader N, Noone AM, Krapcho M, Neyman N, Aminou R, Altekruse SF, et al (eds). SEER Cancer Statistics Review, 1975-2009 (Vintage 2009 Populations). National Cancer Institute [Internet]. Bethesda: National Cancer Institute [citado el 22 de diciembre del 2012]. Disponible en: http://seer.cancer.gov/ csr/1975_2009_pops09/

13. Pow-Sang M, Benavente V, Morante C, Meza L, Olaechea C. Diagnóstico del cáncer de próstata mediante tacto rectal, antígeno prostático específico y biopsia transrectal por ultrasonografía en el Instituto de Enfermedades Neoplásicas (Lima, Perú). Urología Panamericana. 2001;13:35-8.

14. Decreto Supremo $N^{\circ}$ 009-2012-SA. Declaran de interés nacional la Atención Integral del Cáncer y Mejoramiento del Acceso a los Servicios Oncológicos en el Perú y dictan otras medidas. Lima, Perú, 03 de noviembre del 2012. Disponible en: http://www.sisol.gob.pe/home/ boletin-institucional/pdf/BoletinN64/ nl-03-11-12.pdf

15. Resolución Ministerial $\mathrm{N}^{\circ}$ 325-2-12-MINSA. Aprueban el listado de enfermedades de alto costo de atención. Lima, Perú, 24 de abril del 2012. Disponible en: http://censopascindoc. wordpress.com/2012/12/28/resolucion-ministerial-no-325-2012-minsaaprueban-el-listado-de-enfermedadesde-alto-costo-de-atencion/

16. Thompson IM, Ankerst DP, Chi C, Lucia MS, Goodman PJ, Crowley $\mathrm{JJ}$, et al. Operating characteristics of prostate-specific antigen in men with an initial PSA level of $3.0 \mathrm{ng} / \mathrm{ml}$ or lower. JAMA. 2005;294(1):66-70.

17. Greene KL, Albertsen PC, Babaian RJ, Carter HB, Gan PH, Han M, et al. Prostate specific antigen best practice statement: 2009 update. J Urol. 2013;189(1 Suppl):S2-S11.

18. Carter HB, Pearson JD, Metter EJ, Brant LJ, Chan DW, Andres R, et al. Longitudinal evaluation of prostate specific antigen levels in men with and without prostate disease. JAMA. 1992;267(16):2215-20.

19. Loeb S, Metter EJ, Kan D, Roehl KA, Catalona WJ. Prostate-specific antigen velocity (PSAV) risk count improves the specificity of screening for clinically significant prostate cancer. BJU Int. 2012;109(4):508-13.

20. Catalona WJ, Partin AW, Slawin KM, Brawer MK, Flanigan RC, Patel A, et al. Use of the percentage of free prostate-specific antigen to enhance differentiation of prostate cancer from benign prostatic disease: a prospective multicenter clinical trial. JAMA. 1998;279(19):1542-7.

21. Catalona WJ, Richie JP, Ahmann FR, Hudson MA, Scardino PT, Flanigan $\mathrm{RC}$, et al. Comparison or digital rectal examination and serum prostate specific antigen in the early detection of prostate cancer: results of a multicenter clinical trial of 6,630 men. J Urol. 1994;151(5):1283-90.

22. Mistry K, Cable G. Meta-analysis of prostate-specific antigen and digital rectal examination as screening tests for prostate carcinoma. J Am Board Fam Med. 2003;16(2):95-101.

Correspondencia: Mariela Pow Sang

Dirección: Av. Angamos Este 2520. Lima 34, Perú.

Teléfono: (511) 2016500 Anx. 2240

Correoelectrónico:mrpowsang@hotmail.com 